\title{
Bexarotene derivatives modify responses in acute myeloid leukemia
}

Gayla Hadwiger ${ }^{1}$, Orsola di Martino ${ }^{1}$, Margaret A. Ferris ${ }^{2}$, Anh Vu, ${ }^{1}$ Thomas E. Frederick, $^{3}$ Gregory R. Bowman, ${ }^{3}$ Peter Ruminski, ${ }^{1}$ Carl Wagner, ${ }^{4}$ John S. Welch* ${ }^{1}$

${ }^{1}$ Department of Internal Medicine, Washington University, St Louis, Missouri, 63110, USA

2 Department of Pediatrics, Washington University, St Louis, Missouri, 63110, USA

3 Department of Biochemistry and Molecular Biophysics, Washington University, St Louis, Missouri, 63110 USA

${ }^{4}$ School of Mathematical and Natural Sciences, Arizona State University, Phoenix, Arizona, 85281 USA

${ }^{*}$ Corresponding Author: jwelch@wustl.edu

Key words: Retinoid, RXRA, acute leukemia

Running title: Bexarotene derivatives in AML

Abstract: 154 words

Body: 3136 words

Figures: 5

Tables: 0

Supplemental Figures: 2

Supplemental Tables: 1

References: 24 


\section{Abstract}

The retinoids all-trans retinoic acid (ATRA) and bexarotene are active in acute myeloid leukemia (AML), but responses beyond acute promyelocytic leukemia (APL) have been more modest than APL responses. To determine whether chemical modification of bexarotene might augment retinoid responses in AML, we screened a series of 38 bexarotene derivatives for activity in a mouse MLL-AF9 leukemia cell line, which exhibits strong synergistic sensitivity to the combination of ATRA and bexarotene. We found that RXRA potency correlated with antileukemic activity and that only one compound (103-4) with dual RARA/RXRA activity was capable of ATRA-independent anti-leukemic activity. We evaluated bioisostere and cyclohexane modifications for potential resistance to P450 metabolism and found that bioisosteres reduced potency and that bezopyran, cyclopentane, and cyclohexene modifications only modestly reduced susceptibility to metabolism. Collectively, these studies provide a map of the structureactivity relationships of bexarotene with outcomes related to RXRA and RARA activity, corepressor binding, compound stability, and anti-leukemic potential. 


\section{Background}

The retinoic acid receptors (RAR) and retinoid $X$ receptors (RXR) are ligand-activated transcription factors that influence hematopoietic stem cell self-renewal and differentiation (reviewed in [1]). In normal hematopoiesis, RXRA and RARA are dynamically regulated during myeloid maturation, with highest mRNA expression in mature myeloid cells,[2] and have essential roles in granulocyte, macrophage, and osteoclast development.[3, 4, 5, 6] In acute myeloid leukemia (AML) RARA and RXRA have parallel expression among AML subtypes, with highest expression in M4/M5 myelomonocytic and monocytic subtypes.[7, 8]

Transcriptional activation or transcriptional repression by the retinoid receptors is highly dependent on the presence or absence of an activating ligand.[1, 9] RARs function as obligate heterodimers with RXRs, whereas RXRs can function either as a homodimer, or as a heterodimer with other orphan nuclear receptors (e.g. peroxisome proliferator-activated receptors (PPARs), liver $X$ receptor (LXRs), etc). The RARA:RXRA heterodimer binds retinoic acid receptor response elements (RARE); in the absence of an activating ligand, the heterodimer is bound with a co-repressor and ligand binding induces conformational changes that displace the co-repressor and facilitate co-activator binding.[10]

ATRA and bexarotene have been explored in multiple clinical trials in non-APL forms of AML, which repeatedly suggest activity (reviewed in [11]). However, effects have generally been modest. For example, a recent study observed a median overall survival of 8.2 months in AML patients treated with ATRA + decitabine vs 5.1 months in patients receiving decitabine alone ( $p$ $<0.006$ ), and studies using cytotoxic chemotherapy found a median overall survival of 11.3 months with the addition of ATRA vs 7.0 months without.[11-13] Likewise, bexarotene can induce maturation in AML, but clinical responses have been limited.[11, 14, 15] Subsets of patients may exhibit increased sensitivity to retinoids, and biomarker-driven trials are ongoing.[11, 15]

To understand whether subtle chemical changes to bexarotene moieties might increase retinoid anti-leukemic activity achieved during RXRA activation, we evaluated a series of 38 bexarotene derivatives across assays of RXRA activation, RARA activation, anti-leukemic effects with and without concurrent ATRA, ability to release co-repressors from RARA:RXRA heterodimers, and stability of the compounds when co-cultured with HepG2 liver cells. These results provide insight into retinoid structure-activity relationships in AML cells.

\section{Results.}

Structure and activation of $R X R A$.

We characterized a series of 38 bexarotene derivatives for activation of RXRA in mouse MLLAF9-derived primary leukemia cells. These cells were derived by transducing UAS-GFP Kit+ bone marrow cells[16] with MSCV-MLL-AF9. Once leukemia was established in vivo, these cells were harvested and transduced with MSCV-Gal4-RXRA-IRES-mCherry. When treated with an active RXRA ligand, mCherry+ cells become GFP+ (Schema, Figure 1A). We noted a distribution of responses, both in the $\mathrm{EC}_{50}$ of $\mathrm{RXRA}$ reporter activation and in the plateau of the median fluorescence intensity (MFI), a measure of GFP output from the reporter on an individual cell basis (Figure $1 \mathrm{~A}$ and Supplemental Figure $1 \mathrm{~A}-\mathrm{C}$ ). $\mathrm{EC}_{50}$ measured via \%GFP or via GFP MFI correlated closely $\left(\mathrm{R}^{2}=0.97\right)$. The $M F I \mathrm{EC}_{50}$ also correlated with maximum $\mathrm{MFI}$, although compounds with high maximal reporter MFI tended to have low potency (i.e. high $\left.E C_{50}\right),\left(R^{2}=0.3\right.$, Figure 1B). 
We had previously observed anti-leukemic synergy between RXRA ligands and RARA ligands.[8] To assess anti-leukemic effects across diverse RXRA compounds, we treated MLLAF9 leukemia cells with increasing concentrations of each compound in the presence of $100 \mathrm{nM}$ ATRA. Anti-leukemic effects were measured as the total number of viable cells after 5 days across a dose dilution to provide an $\mathrm{IC}_{50}$. We observed that the anti-leukemic $\mathrm{IC}_{50}$ correlated with the Gal4-RXRA activation $\mathrm{EC}_{50}$ of the compound $\left(\mathrm{R}^{2}=0.97\right)$, and there was less correlation with the maximum MFI $\left(R^{2}=0.27\right)$ (Figure $\left.1 C\right)$. We generated an MLL-AF9 leukemia line that was resistant to ATRA and bexarotene after culturing the cells in $100 \mathrm{nM}$ ATRA/bexarotene for 6 weeks. We reassessed growth after 5 days of ATRA $100 \mathrm{nM}$ and $250 \mathrm{nM}$ of each compound to determine whether resistance was acquired generally to the compounds or whether resistance was variable across compounds. The number of viable cells after 5 days in culture correlated with the initial $I_{50}$, suggesting global mechanisms of resistance rather than compound-specific sensitivity mechanisms (Figure 1D).

Within the compound set, series of molecularly related molecules could be identified for structure-activity relationships (Figure 1E-G). In general, we found that replacement of the carbon atom at $\mathrm{C} 6$ in the 5,6,7,8-tetrahydro-3,5,5,8,8-pentamethyl-2-naphthalenyl part of the bexarotene molecule with oxygen, to form the 3,4-Dihydro-1,1,4,4,7-pentamethyl-1H-2benzopyran analogue, reduced both the potency and the anti-leukemic activity (i.e. they increased the GFP reporter $\mathrm{EC}_{50}$ and the leukemic viability $\left.\mathrm{IC}_{50}\right)$. In bexarotene, the two benzene rings are connected by a linker carbon with an alkenyl group. Substituting this alkenyl group at the linker carbon with a cyclopropyl group or replacing the carbon linker with an aliphatic substituted nitrogen as the linker (with ethyl and allyl substitution being preferred), increased both the potency and the anti-leukemic activity. Likewise, across multiple compounds, replacing the benzoic acid portion of bexarotene with nicotinic acid or a pyrimidine acid were associated with increasing potency and anti-leukemic activity.

Repeat assessments of highly potent compounds validated a series of compounds with increased potency compared with bexarotene or fluoro-bexarotene (fBex) (Supplemental Figure $1)$. Replicate results correlated with initial results $\left(R^{2}=0.83\right)$. A range of potency was noted, with the most potent compounds having similar activity as LG268. Anti-leukemic activity again correlated with RXRA activation potency $\left(R^{2}=0.71\right.$, Supplemental Figure 1D). Low $n M E_{50}$ was validated across this compound set using a second reporter assay (293T cells transfected with ApoA-Luciferase and RXRA, Supplemental Figure 1E).

\section{Structure and activation of RARA}

We assessed all compounds for the ability to cross-activate a UAS-GFP/Gal4-RARA reporter in MLL-AF9 leukemia cells (Schema, Figure 2A). We noted several compounds with low potency RARA activation and one compound (103-4) with potent dual RXRA and RARA activity (Figure 2A, 103-4 EC $\mathrm{EC}_{50}$ Gal4-RXRA: $14 \mathrm{nM}$; Gal4-RARA: $\left.15 \mathrm{nM}\right)$. We determined the anti-leukemic activity of each compound in the presence and absence of concurrent ATRA, noting that 103-4 uniquely was capable of inhibiting leukemic growth independent of concurrent ATRA treatment (Figure 2B).

RARA:RXRA forms a heterodimer, and the components of the dyad differentially interact with the corepressors NCOR1 (Nuclear Receptor Corepressor 1, formerly NCOR) and NCOR2 (Nuclear Receptor Corepressor 2, formerly SMRT).[17] We used a mammalian two-hybrid assay to determine whether different RXRA ligands differentially released co-repressors bound to RARA:RXRA heterodimers. Compound effects with Gal4-NCOR1 and RARA:RXRA correlated with effects with Gal4-NCOR2 (Figure $2 C, R^{2}=0.68$ ). ATRA and 103-4 induced the greates co-repressor release from RARA:RXRA (Figure $2 \mathrm{C}$ ), and these results correlated with 
activation of the Gal4-RARA reporter assay (Figure 2D: Gal4-NCOR1 $\mathrm{R}^{2}=0.64$; Gal4-NCOR2 $\left.R^{2}=0.61\right)$. Thus, 103-4 uniquely exhibited both co-repressor released from RARA:RXRA and anti-leukemic activity in the absence of ATRA (Figure 2E-F).

We used in silico docking algorithms to assess the predicted binding of the compounds to RARA crystal structures generated in the presence of an agonist (AM580: PDB 3KMR) or an antagonist (BMS493: 3KMZ). We observed no correlation between predicted docking scores and RARA activation or co-repressor release (Supplemental Figure 2). The docking scores for the average and maximum compound conformation correlated modestly between $3 \mathrm{KMR}$ and $3 \mathrm{KMZ}\left(\mathrm{R}^{2}=0.36\right.$ and $R^{2}=0.22$, respectively).

\section{Prior dual RARA:RXRA ligands}

Because 103-4 exhibited unique single-agent anti-leukemic activity, we evaluated the activity of two additional ligands with reported dual affinity. Compound $15 \mathrm{~b}$ and 4-\{4-[3(trifluoromethyl)phenyl]-1,3-thiazol-2-yl\}benzoic acid (PTB) had been previously suggested to maintain some dual affinity activity.[18, 19] In our assays, $15 \mathrm{~b}$ was a potent RARA ligand, but had little RXRA activation, and was not capable of anti-leukemic activity alone or in combination with ATRA (Figure 3A-D). PTB was a weak RARA and RXRA agonist, and exhibited no antileukemic activity as a single agent (Figure $3 E-H)$.

\section{Circumventing Metabolism}

Bexarotene has a short serum half-life, in part, due to hepatic metabolism. 6- and 7-oxo and 6and 7-hydroxy bexarotene and the corresponding hydroxylglucuronides are major metabolites in human, with glucuronidation of the carboxylic acid (acyl glucuronide) identified as metabolites as well.[20] Acid bioisosteres are simple chemical modifications of carboxylic acids that have the potential to block acyl glucuronidation while preserving the binding functionality of the free acid. Converting the benzoic acid of bexarotene to a hydroxamic acid provides the possibility of continued ionic interactions with the essential R321 that usually docks with the carboxylic acid. We synthesized a series of 3 bioisosteres (Figure 4A).[21] In silico docking experiments suggested variable interference with ligand binding to two different RXRA configurations (Figure 4B-C). However, all of these modifications reduced the potency RXRA activation and antileukemic activity of the resultant compounds (Figure 4D-E).

To determine whether modifications at the C6 carbon might reduce hepatic inactivation of bexarotene, we screened the effect of HepG2 co-culture on ligand stability, comparing six such C6 modified analogs against bexarotene. Each compound was cultured for 48 or 96 hours in media alone, with HepG2 cells, or HepG2 cells that stably overexpress CYP3A4. Media was then collected and assayed for RXRA ligand activity using RXRA reporter cells (schema Figure $5 A-B)$. The six bexarotene derivates were selected with pairwise alterations: replacing the 5,6,7,8-tetrahydro-3,5,5,8,8-pentamethyl-2-naphthalenyl part of the bexarotene molecule with the 5-membered 2,3-Dihydro-1,1,3,3-tetramethyl-1H-indene (003-6 and 007-6); replacing the C6 carbon of the 5,6,7,8-tetrahydro-3,5,5,8,8-pentamethyl-2-naphthalenyl part of the bexarotene with an oxygen to form a 3,4-Dihydro-1,1,4,4,7-pentamethyl-1H-2-benzopyran (175-6 and 1696 ); or by forming an unsaturated bond between the C5 and C6 carbons of the 5,6,7,8tetrahydro-3,5,5,8,8-pentamethyl-2-naphthalenyl part of the bexarotene molecule to form a 1,4Dihydro-1,1,4,4-tetramethylnaphthalene (103-4 and 149-3).

All six of these compounds retained good $R X R$ activation and anti-leukemic function (Supplemental Table 1). We noted very little reduction in RXRA activity following 96 hours of incubation in cell culture media at 37 degrees, suggesting that these compounds are remarkably stable (Figure 5C). Compared to bexarotene, the two compounds with the 5-membered 2,3- 
Dihydro-1,1,3,3-tetramethyl-1H-indene group (003-6 and 007-6) had increased sensitivity to HepG2A metabolism, the 3,4-Dihydro-1,1,4,4,7-pentamethyl-1H-2-benzopyran analogs had similar sensitivity (175-6 and 169-6), and the unsaturated 1,4-Dihydro-1,1,4,4tetramethylnaphthalene analogues (103-4 and 149-3) had modestly reduced sensitivity, suggesting that they might have longer serum half-lives (Figure 5D-E).

\section{Discussion}

The pan-RAR agonist all-trans retinoic acid (ATRA, tretinoin) transformed the treatment of APL, and has shown reproducible, but more limited activity in non-APL AML.[11-13] Bexarotene is approved for the treatment of CTCL, is also tolerable in AML patients, and has demonstrated some activity in AML, with evidence of maturation effects,[11, 14, 15] again suggesting that retinoids might be therapeutically relevant in non-APL AML.

We sought to characterize a series of bexarotene derivatives and to define their structureactivity relationships. We used a UAS/Gal4 reporter system in primary mouse leukemia cells to assess RXRA activation. The reporter reads out three parameters: percent GFP+ cells, the median fluorescence intensity of GFP among the GFP+ cells (MFI), and the plateau of the GFP MFI. When a series of dose dilutions are used, $\mathrm{EC}_{50}$ is calculated for the first two parameters. The \%GFP+ $\mathrm{EC}_{50}$ and the MFI EC $\mathrm{E}_{50}$ correlated closely (Supplemental Figure 1C). The plateau of the GFP MFI also directly correlated with the \%GFP EC EO (Figure 1B), suggesting that less potent compounds could achieve greater GFP reporter output. Anti-leukemic $\mathrm{IC}_{50}$ correlated with RXRA potency (low EC $\mathrm{E}_{50}$ ), not with the plateau of the GFP MFI, perhaps because less potent compounds are less toxic, and the reporter is able to achieve greater per-cell-output when exposed to compounds with low potency (i.e. high $\mathrm{EC}_{50}$ ).

Using this approach, we correlated structure and activity, noting that improved potency and antileukemic activity correlated with longer linker side chains, pyridine rings, and inclusion of the benzene methyl group (Figure 1).

Across this set of compounds, we noted several compounds with weak RARA activity and one compound with strong dual affinity for RARA and RXRA (Figure 2A, 103-4). Activation of RARA correlated with the release of the co-repressors NCOR1 and NCOR2 from RARA:RXRA heterodimers in a mammalian two-hybrid assay (Figure 2C-D). ATRA-independent antileukemic activity was exhibited by only one compound (103-4). This compound also exhibited RARA potency and the ability to release co-repressors from RARA:RXRA heterodimers, suggesting the potential necessity of RARA activation and/or co-repressor release to achieve maximum retinoid-induced anti-leukemic activity. Thus, co-repressor release from the RARA:RXRA heterodimer appears to be determined more by RARA activation than RXRA activation, consistent with prior data suggesting a subordinate role of RXRA in the RXRA:RARA heterodimer.[22, 23]

Bexarotene has a short serum half-life, in part due to hepatic metabolism.[20] We generated a series of bioisosteres to potentially block glucuronidation, and we screened compounds with modifications at the cyclohexane $\mathrm{C} 6$ for protection from hepatic metabolism. The bioisosteres all had marked reduction in $R X R A E_{50}$, likely due to steric hindrance at R321, a necessary anionic interaction to effectively dock the ligand in the ligand-binding pocket.[21] WU0087 was developed using fluorobexarotene as the backbone and proved particularly non-functional, suggesting that intramolecular interactions between the fluoro and the carbonyl groups may have formed bulky steric inhibition of the necessary interactions with R321 or other amino acid 
groups. Use of the more potent LG268 backbone may have helped overcome some of the detrimental effects of the bioisostere, relative to the bexarotene backbone, but this was not sufficient to generate a potent, active ligand (Figure 4).

We compared stability in tissue culture of 6 compounds against bexarotene and found that they exhibited remarkable stability in tissue culture media at $37^{\circ}$ over 96 hours (Figure $5 \mathrm{C}$ ). The substitution of a cyclohexene for cyclohexane modestly reduced HepG2 metabolism, whereas substitution of a cyclopentane or oxocyclohexane ring resulted in similar or more rapid metabolism by HepG2 cells (Figure 5) as well as reduced activity (Figure 1). However, these limited improvements in resistance to HepG2 metabolism are not likely to have large effects on the in vivo pharmacokinetics of bexarotene.

Collectively, these studies provide structure-activity relationships for bexarotene and a series of derivatives. Correlations across this broad set of chemicals demonstrate a strong relationship between potency in receptor activation assays and anti-leukemic activity, and that modifications to bexarotene can improve both the RXRA potency and the retinoid anti-leukemic activity.

\section{Materials and Methods}

Reagents. Bexarotene was from LC Laboratories. ATRA and PTB were from Sigma-Aldrich. Bioisostere and 15b compounds were synthesized by WuXi. Cytokines were purchased from R\&D Systems. The Gal4-NCOR2, Gal4-NCOR1, NCOR2-VP16, NCOR1-VP16, VP16-RARA, and VP16-RXRA plasmids were gifts from Mitch Lazar, University of Pennsylvania. The pBABERXRA and ApoA1-Luciferase plasmids were gifts from Vivek Arora, Washington University. HepG2-EV and HepG2-3A4 cells were provided by the Division of Biochemical Toxicology, National Center for Toxicological Research.

Compound synthesis. All other compounds were synthesized in the Carl Wagner laboratory.

Generation of MLL-AF9 mouse leukemia. Mouse bone marrow Kit+ cells were isolated from UAS-GFP mice using an Automacs Pro (Miltenyl Biotec, San Diego, CA) per the manufacture's protocol. Kit+ cells were plated in progenitor expansion medium (RPMI1640 medium, 15\% FBS, Scf $(50 \mathrm{ng} / \mathrm{ml})$, IL3 $(10 \mathrm{ng} / \mathrm{ml})$, Flt3 $(25 \mathrm{ng} / \mathrm{ml})$, Tpo $(10 \mathrm{ng} / \mathrm{ml})$, L-glutamine $(2 \mathrm{mM})$, sodium pyruvate $(1 \mathrm{mM})$, HEPES buffer $(10 \mathrm{mM})$, penicillin/streptomycin $(100 \mathrm{units} / \mathrm{ml}), \quad \beta-$ mercaptoethanol $(50 \mu \mathrm{M})$ ) overnight and transduced by spinfection with $10 \mu \mathrm{g} / \mathrm{ml}$ polybrene and $10 \mathrm{mM}$ HEPES at $2400 \mathrm{rpm}, 30^{\circ} \mathrm{C}$ for 90 minutes in an Eppendorf $5810 \mathrm{R}$ centrifuge. Cells were transplanted into sublethally irradiated mice and subsequent leukemia harvested 2-4 months later, as expected.[24] MLL-AF9 leukemia cells were cultured in vitro using similar media, but without Flt3, or Tpo. Fluorescence was detected on an Attune NxT Flowcytometer (Invitrogen) or ZE5 Cell Analyzer (Biorad).

Mice. UAS-GFP mice were bred as described.[6, 16] The Washington University Animal Studies Committee approved all animal experiments.

\section{Retrovirus production}

Retrovirus production was performed as described using previously described vectors.[6, 16] 7 $x 10^{6} 293 \mathrm{~T} / 17$ cells were seeded in a $150 \mathrm{~cm}^{2}$ dish in DMEM (high glucose) $+10 \% \mathrm{FBS}+1 \%$ Glutamax, 18-24 hours before transfection and grown to $80 \%$ confluence. $30 \mu \mathrm{g}$ of DNA, 21.5 $\mu \mathrm{g}$ Ecopak, and $1.25 \mathrm{~mL}$ of DMEM were mixed. $40 \mu \mathrm{L}$ Lipofectamine $3000+1.25 \mathrm{~mL}$ DMEM were mixed. The two mixtures were incubated for 5 minutes, then mixed together and incubated for 15 minutes. $1.25 \mathrm{~mL}$ of the mixture were dropped-wise onto the $293 \mathrm{~T} / 17$ cells. 
Fresh medium was changed after 18-24 hours transfection. Virus was collected at 48 hours and 72 hours and concentrated with Lenti-X Concentrator (Clontech). The Virus was resuspended in DMEM and stored at $-80 \mathrm{C}$.

$\mathbf{E C}_{50}$ and $\mathbf{I C}_{50}$ calculations. UAS-GFP/MLL-AF9 leukemia cells were transduced with either MSCV-3xFlag-Gal4 (DBD)-RXRA (LBD)-IRES-mCherry or MSCV-Gal4 (DBD)-RARA (LBD)IRES-mCherry. Typically, compounds were diluted in $100 \mu \mathrm{ls}$ in 96 well plates in duplicate, starting at $1 \mu \mathrm{M}$ through $121: 1$ dilutions. Cells were added in $100 \mu \mathrm{l}$ media aliquots and then evaluated by flow cytometry 48 hours later. Cell viability was similarly plated on day 1 . On day 3 , $10 \mu \mathrm{l}$ were replated into freshly diluted compounds and new media. On day 5 total viable cells per well were evaluated by flow cytometry. $\mathrm{EC}_{50}$ and $\mathrm{IC}_{50}$ were calculated using Prism Graphpad.

Mammalian two-hybrid assay. 293Tcells were co-transfected using Lipofectamine 3000 (Invitrogen) with plasmids encoding the reporter: UAS-GFP; Gal4-fusion vectors as "bait"; and VP16-fusions as "prey". The percentage of GFP+ cells was assessed 48 hours after transfection by flow cytometry.

Luciferase detection. 293T cells were transfected with pBABE-RXRA in combination with ApoA1-Luciferase using Lipofectamine 2000 (Invitrogen). Six hours after transfection, the cells were collected and plated into a 48 well plate in 1\% BSA media and treated in triplicate. After 40 hours incubation, the cells were harvested and assayed for luciferase (Luc Assay System with Reporter Lysis Buffer, Promega) in a Beckman Coulter LD400 plate reader.

\section{HepG2 analysis.}

HepG2 cells that stably express cytochrome P450s (CYPs) and a control, empty vector (EV), with a Blasticidin marker were grown in the recommended media (HepG2 Media: Gibco High Glucose DMEM with 10\% FBS, 100U/mL Pen/Strep, 0.25 ug/mL Blasticidin, $1 \%$ Glutamax, 1 mL NEAA (non-essential amino acids)). The cells were plated onto a 12 well plate @ 500,000 cells per well in $0.5 \mathrm{~mL}$ media and grown overnight at $37^{\circ} \mathrm{C}$ in a tissue culture $\mathrm{CO} 2$ incubator. The next day, the media was replaced with new media, without Pen/Strep, and drug dilutions were added. Media for each drug dilution was collected from the cells at 0, 48, and 96 hours and frozen @ $-80^{\circ} \mathrm{C}$. UAS-GFP MLL-AF9 reporter cells were plated onto a 96 well plate at 10,000 cells per well in $0.1 \mathrm{~mL}$ media from the thawed HepG2 metabolized drug aliquots via serial dilutions. After 48 hours, the cells were analyzed on an Invitrogen Attune NxT flow cytometer.

Compound docking. Analysis was performed with Surflex-dock (26). MOL2 files for each compound were generated from SMILES strings using Open Babel.(41) Surflex-Dock receptor protomols were generated with a threshold of 0.25 and a bloat of 2.0 and subsequently docked using the default '-pgeom' docking accuracy parameter set.

\section{Data analysis}

Flow cytometry data was analyzed with FlowJo software version 10. Statistical analysis was performed using Prism (Graphpad). T-test and ANOVA tests were performed, as appropriate. Error bars represent standard deviation. Data points without error bars have standard deviations below Graphpad's limit to display.

Acknowledgments: We thank the Alvin J. Siteman Cancer Center at Washington University School of Medicine and Barnes-Jewish Hospital in St. Louis, MO. for the use of the Flow 
Cytometry Core. The Siteman Cancer Center is supported in part by an NCl Cancer Center Support Grant P30 CA91842. We thank Conner York for technical assistance. This work was supported by NIH R01 HL128447 (JS Welch), by the Siteman Investment Program (JS Welch), the Washington University SPORE DRP (JS Welch), and the Children's Discovery Institute (JS Welch). Author Contributions: J.S.W. and G.H. designed experiments, performed experiments, and wrote the manuscript. A.V., O.d.H, M.A.F., T.E.F, G.R.B, P.R. designed and performed experiments. Compounds were synthesized by C.W.

1. Oren, T., J.A. Sher, and T. Evans, Hematopoiesis and retinoids: development and disease. Leuk Lymphoma, 2003. 44(11): p. 1881-91.

2. $\mathrm{Niu}, \mathrm{H}$. , et al., Endogenous retinoid $X$ receptor ligands in mouse hematopoietic cells. Sci Signal, 2017. 10(503).

3. Purton, L.E., et al., RARgamma is critical for maintaining a balance between hematopoietic stem cell self-renewal and differentiation. J Exp Med, 2006. 203(5): p. 1283-93.

4. Kizaki, M., et al., Effects of novel retinoid $X$ receptor-selective ligands on myeloid leukemia differentiation and proliferation in vitro. Blood, 1996. 87(5): p. 1977-84.

5. Taschner, S., et al., Down-regulation of RXRalpha expression is essential for neutrophil development from granulocyte/monocyte progenitors. Blood, 2007. 109(3): p. 971-9.

6. Menendez-Gutierrez, M.P., et al., Retinoid X receptors orchestrate osteoclast differentiation and postnatal bone remodeling. J Clin Invest, 2015. 125(2): p. 809-23.

7. Welch, J.S., et al., A phase I dose escalation study of oral bexarotene in combination with intravenous decitabine in patients with AML. Am J Hematol, 2014. 89(8): p. E103-8.

8. Di Martino, $\mathrm{O}$., et al., Endogenous and combination retinoids are active in myelomonocytic leukemias. Haematologica, 2021. 106(4): p. 0.

9. Kastner, P., et al., Positive and negative regulation of granulopoiesis by endogenous RARalpha. Blood, 2001. 97(5): p. 1314-20.

10. Loinder, K. and M. Soderstrom, The nuclear receptor corepressor ( $N$-COR) modulates basal and activated transcription of genes controlled by retinoic acid. J Steroid Biochem Mol Biol, 2003. 84(1): p. 15-21.

11. Martino, O.D. and J.S. Welch, Retinoic Acid Receptors in Acute Myeloid Leukemia Therapy. Cancers (Basel), 2019. 11(12).

12. Lubbert, M., et al., Valproate and Retinoic Acid in Combination With Decitabine in Elderly Nonfit Patients With Acute Myeloid Leukemia: Results of a Multicenter, Randomized, 2 × 2, Phase II Trial. J Clin Oncol, 2019: p. JCO1901053.

13. Schlenk, R.F., et al., Phase III study of all-trans retinoic acid in previously untreated patients 61 years or older with acute myeloid leukemia. Leukemia, 2004. 18(11): p. 1798-803.

14. Welch, J.S., Mutation position within evolutionary subclonal architecture in AML. Semin Hematol, 2014. 51(4): p. 273-81.

15. McKeown, M.R., et al., Super-Enhancer Analysis Defines Novel Epigenomic Subtypes of Non-APL AML Including an RARalpha Dependency Targetable by SY-1425, a Potent and Selective RARalpha Agonist. Cancer Discov, 2017.

16. Niu, H., Chacko, J., Hadwiger, G., Welch, J.S., Absence of natural intracellular retinoids in mouse bone marrow cells and implications for PML-RARA transformation. Blood Cancer J, 2015. 5: p. e284.

17. Cordeiro, T.N., et al., Interplay of Protein Disorder in Retinoic Acid Receptor Heterodimer and Its 
Corepressor Regulates Gene Expression. Structure, 2019. 27(8): p. 1270-1285 e6.

18. Simoni, D., et al., Heterocycle-containing retinoids. Discovery of a novel isoxazole arotinoid possessing potent apoptotic activity in multidrug and drug-induced apoptosis-resistant cells. J Med Chem, 2001. 44(14): p. 2308-18.

19. Koshiishi, C., et al., Identification and characterization of a phenyl-thiazolyl-benzoic acid derivative as a novel RAR/RXR agonist. Heliyon, 2019. 5(11): p. e02849.

20. Howell, S.R., et al., Bexarotene metabolism in rat, dog, and human, synthesis of oxidative metabolites, and in vitro activity at retinoid receptors. Drug Metab Dispos, 2001. 29(7): p. 990-8.

21. Bourguet, W., et al., Crystal structure of a heterodimeric complex of RAR and RXR ligand-binding domains. Mol Cell, 2000. 5(2): p. 289-98.

22. Altucci, L., et al., Rexinoid-triggered differentiation and tumor-selective apoptosis of acute myeloid leukemia by protein kinase A-mediated desubordination of retinoid $X$ receptor. Cancer Res, 2005. 65(19): p. 8754-65.

23. Germain, P., et al., Co-regulator recruitment and the mechanism of retinoic acid receptor synergy. Nature, 2002. 415(6868): p. 187-92.

24. Somervaille, T.C. and M.L. Cleary, Identification and characterization of leukemia stem cells in murine MLL-AF9 acute myeloid leukemia. Cancer Cell, 2006. 10(4): p. 257-68. 
Figure 1. Correlation of compounds' RXRA activity and antileukemic activity. A. Activation of a UAS-GFP/Gal4-RXRA reporter by bexarotene derivatives. B. Correlation of MFI EC $\mathrm{E}_{50}$ and MFI plateau in UAS-GFP/Gal4-RXRA reporter output. C. Correlation of UAS-GFP/Gal4-RXRA reporter activation $\mathrm{EC}_{50}$ and antileukemic activity in MLL-AF9 leukemia cells. D. Correlation of antileukemic activity in parental MLL-AF9 leukemia cells vs. cells selected for retinoid resistance by culturing for 6 weeks in $100 \mathrm{nM}$ bexarotene and $100 \mathrm{nM}$ ATRA. E - G. Structure-activity correlations.

Figure 2. Correlation of compound RARA activity and antileukemic activity. A. Activation of UAS-GFP/Gal4-RARA reporter. B. Anti-leukemic activity of compounds with and without $100 \mathrm{nM}$ ATRA. C. Mammalian two-hybrid analysis of Gal4-NCOR1 and Gal4-NCOR2 interactions with VP16-RARA:VP16-RXRA. 293T cells were transfected with UAS-GFP, VP16-RARA, VP16RXRA, and either Gal4-NCOR1 or Gal4-NCOR2 and GFP assessed with and without compounds. D. Correlation of UAS-GFP/Gal4-RARA activation with corepressor release in mammalian two-hybrid. E - F. Correlation of corepressor release in mammalian two-hybrid assay with antileukemic activity.

Figure 3. Analysis of reported dual affinity ligands. A-C. Compound $15 \mathrm{~b}$ assessed for activation of UAS-GFP/Gal4-RXRA, UAS-GFP/Gal4-RARA, and antileukemic activity. D-F. PTB assessed for activation of UAS-GFP/Gal4-RXRA, UAS-GFP/Gal4-RARA, and antileukemic activity.

Figure 4. Bioisosters. A. Three bioisosteres were generated using bexarotene, fluorobexarotene, and LG268 as backbones. B-C. Docking predictions for RXRA binding using two different available crystal structures. D. Activation of UAS-GFP/RXRA-Gal4 reporter. E - F. Anti-leukemic effects without and with $100 \mathrm{nM}$ ATRA.

Figure 5. Compound metabolism by HepG2 cells. A. Schema. Compounds were added to cell culture media, with and without cells. After 0, 48, and 96 hours, media was collected and dilutions applied to UAS-GFP/Gal4-RXRA reporter cells. GFP was assessed 48 hours later and an AUC calculated. B. Representative AUC calculation. C-E. Normalized AUC when compounds were plated in media alone, with HepG2-EV cells (empty vector), or with HepG2$3 A 4$ cells (stable expression of CYP3A4). F. Structures of compounds assessed.

Supplemental Figure 1. Assessment of compounds with $E C_{50}$ potency greater than bexarotene. A. Activation of UAS-GFP/Gal4-RXRA reporter in MLL-AF9 leukemia cells measured by percent GFP+ cells. B. Activation of UAS-GFP/Gal4-RXRA reporter in MLL-AF9 leukemia cells measured by median fluorescence intensity (MFI) of GFP+ cells. C. Correlation of $\mathrm{EC}_{50}$ calculated by percent GFP+ and by MFI. D. Correlation of antileukemic activity, measured by $\mathrm{IC}_{50}$ of each compound, and the $\mathrm{EC}_{50}$ activation of the UAS-GFP/Gal4-RXRA reporter. E. Activation of a ApoA-luciferace reporter. F. Structures of labeled, potent compounds.

Supplemental Figure 2. Predicted compound binding to RARA. A. Docking scores for compounds bound to 3KMR (RARA bound to the RARA agonist AM580). B. Docking scores for compounds bound to 3KMR (RARA bound to the RARA antagonist BMS493). Each dot represents results for a separate compound conformation. Red indicates compounds with activity in UAS-GFP/Gal4-RARA reporter assay (Figure 2A). 
bioRxiv preprint doi: https://doi.org/10.1101/2021.05.17.444475; this version posted May 17, 2021. The copyright holder for this preprint (which was not certified by peer review) is the author/funder, who has granted bioRxiv a license to display the preprint in perpetuity. It is made available under aCC-BY-NC-ND 4.0 International license.

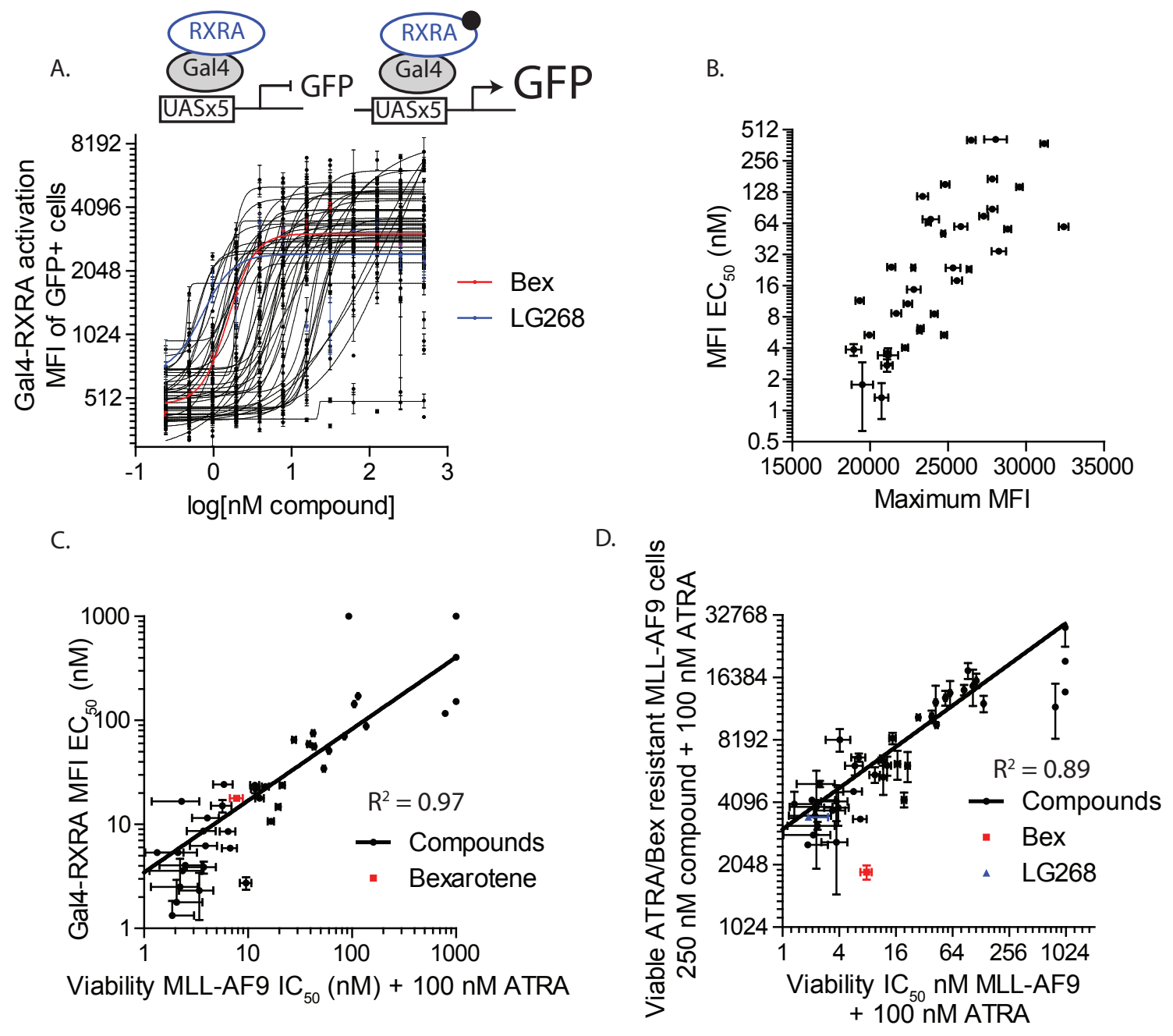

E.

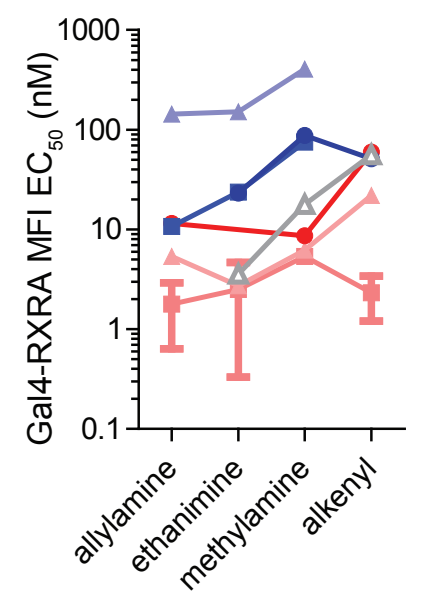

F.

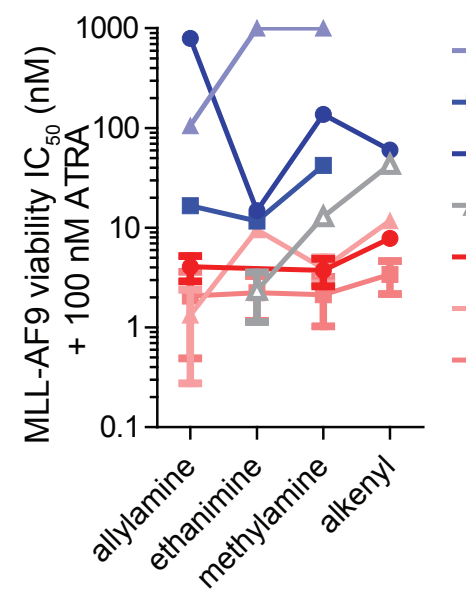

G.<smiles>[Y]c1cc2c(cc1[X])C(C)(C)C[Y]C2(C)C</smiles>

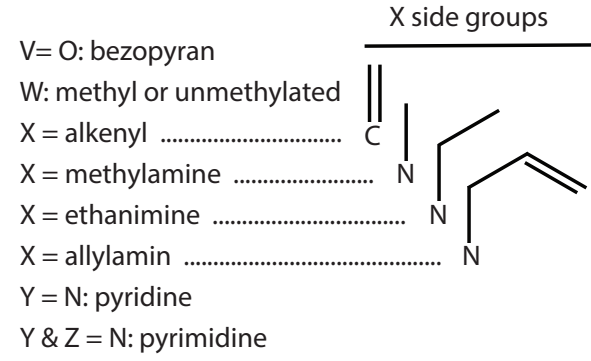

Figure 1. 
A.

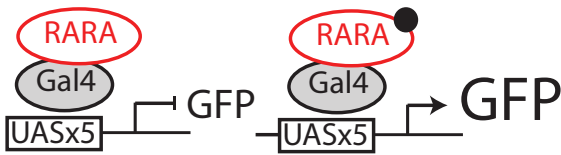

Gal4-RARA

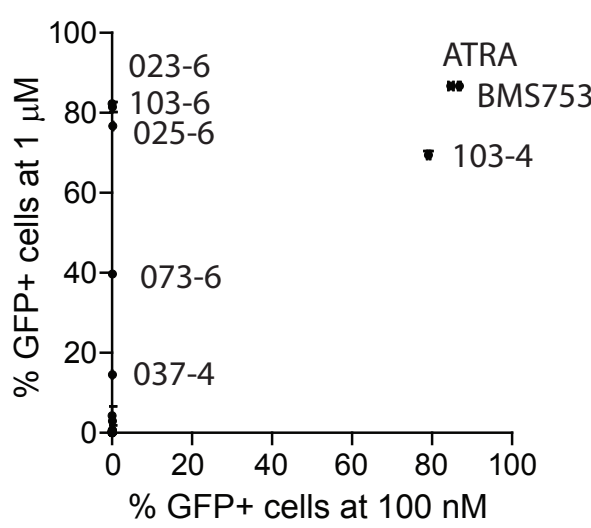

C.

Corepressor release from RXRA:RARA

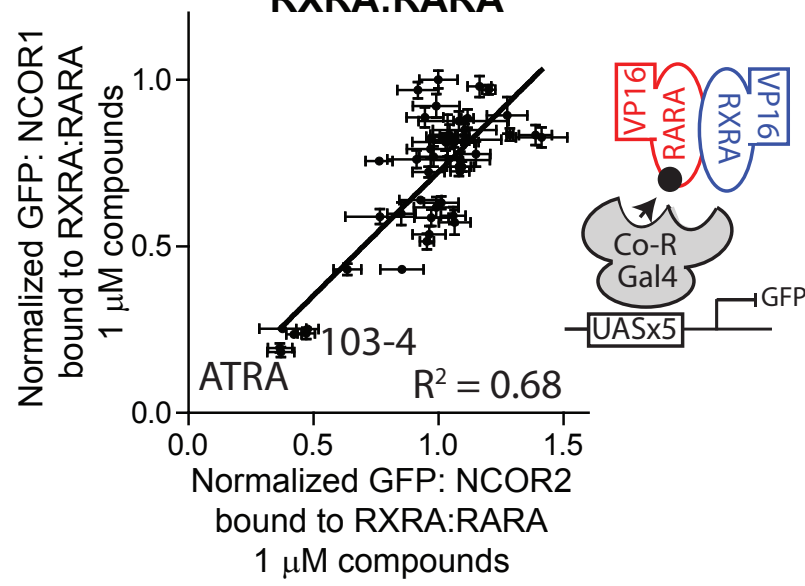

E.

\section{MLLAF9 sensitivity single agent vs corepressor release}

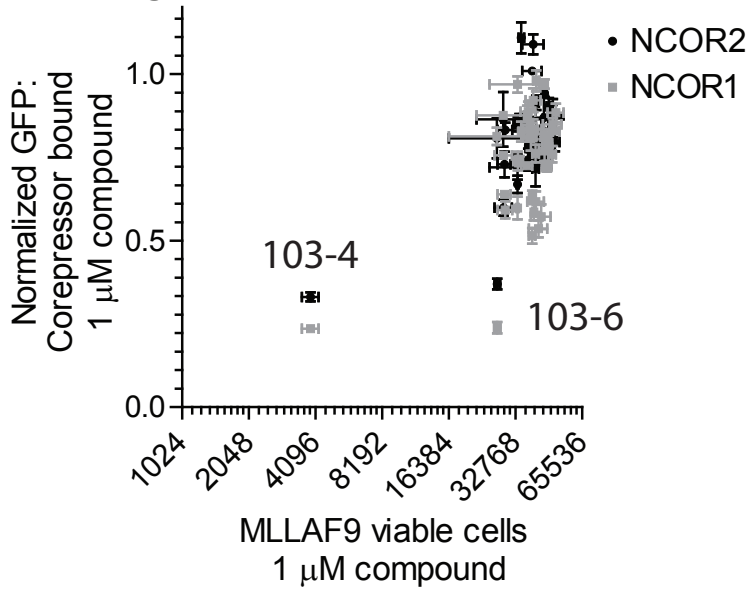

B.

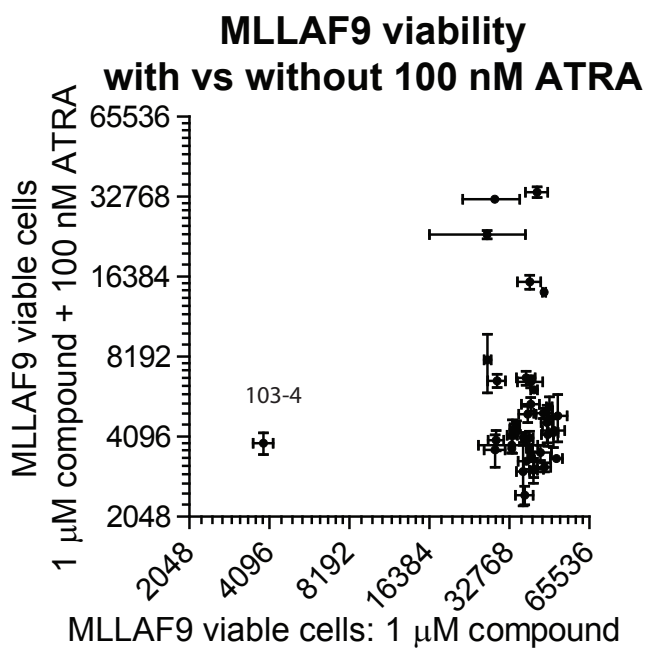

D.

\section{RARA activation vs Corepressor release}

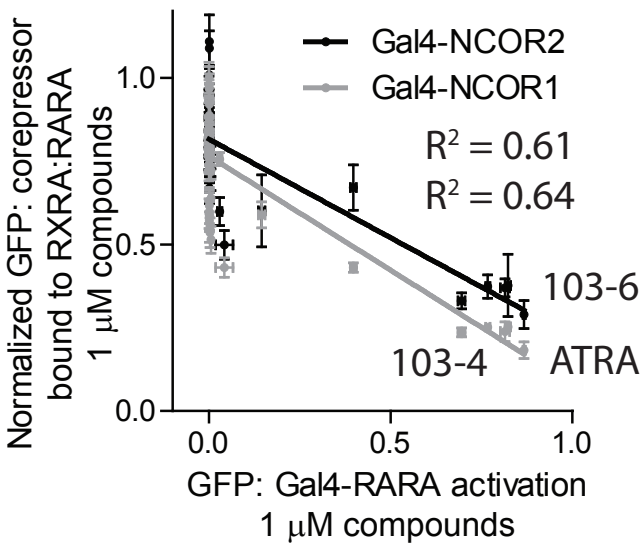

F.

\section{MLLAF9 sensitivity with concurrent ATRA vs corepressor release}

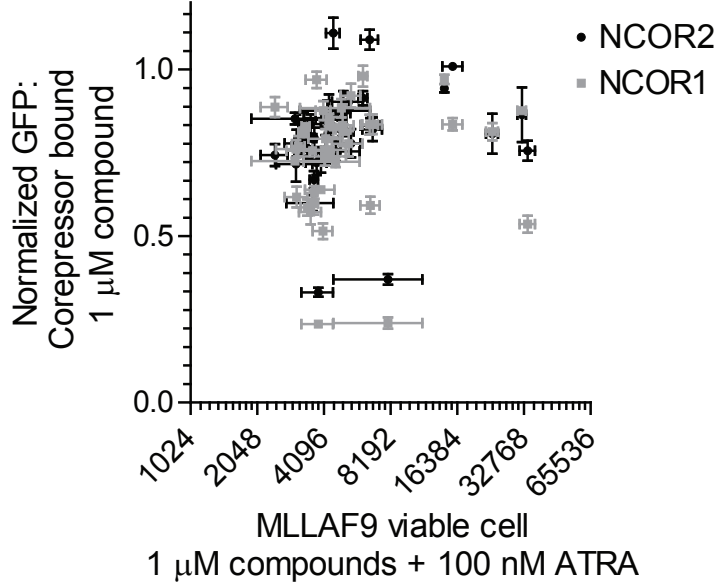

Figure 2. 
A.<smiles>CC1(C)CCC(C)(C)c2cc(-c3cc(-c4ccc(O)cc4)no3)ccc21</smiles>

B.

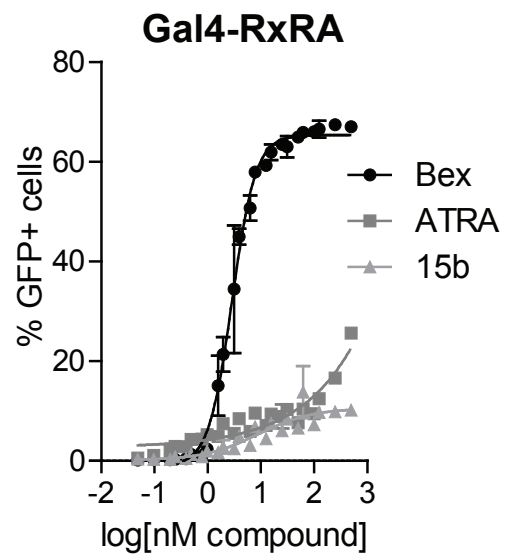

E.<smiles>Oc1ccc(-c2nc(-c3cccc(C(F)(F)F)c3)cs2)cc1</smiles>

F.

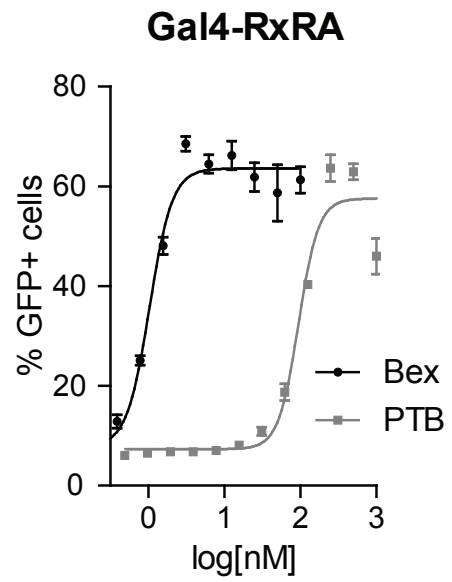

C.

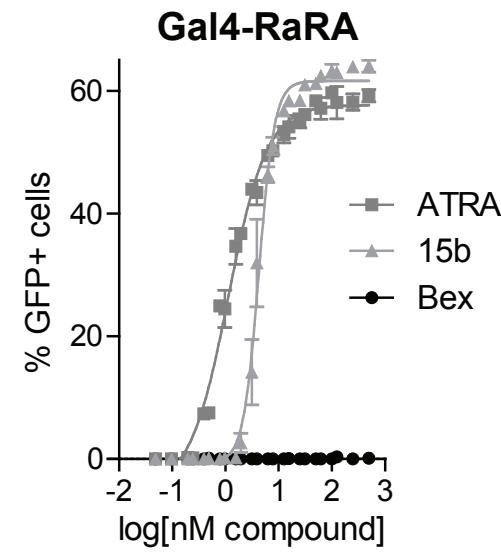

D.

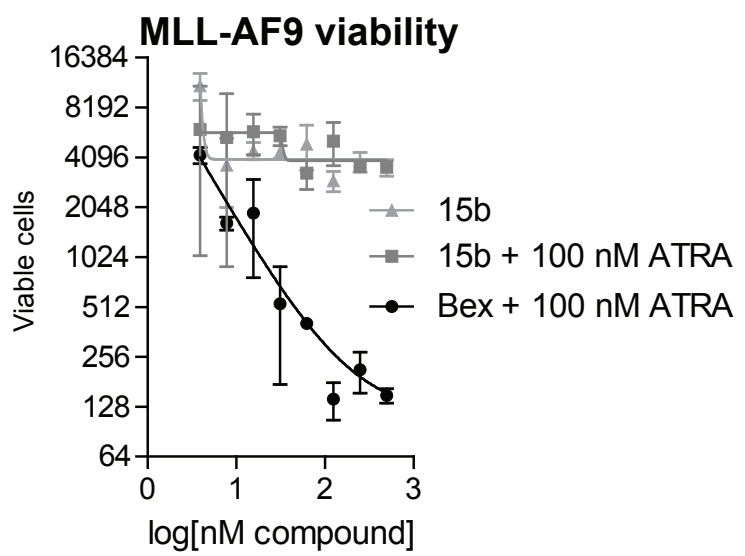

H.

Gal4-RaRA

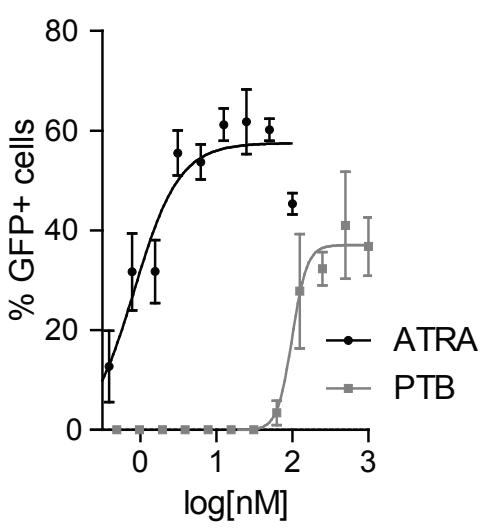

\section{MLL-AF9 viability}

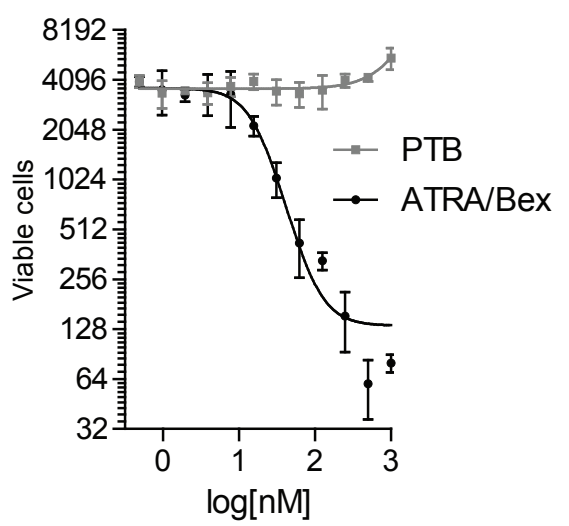

Figure 3. 
A.<smiles>C=C(c1ccc(C(=O)O)cc1)c1cc2c(cc1C)C(C)(C)CCC2(C)C</smiles><smiles>Cc1cc2c(cc1C1(c3ccc(C(=O)NO)cn3)CC1)C(C)(C)CCC2(C)C</smiles><smiles>C=C(c1ccc(C(=O)NO)c(F)c1)c1cc2c(cc1C)C(C)(C)CCC2(C)C</smiles><smiles>C=C(c1ccc(C(=O)NO)cc1)c1cc2c(cc1C)C(C)(C)CCC2(C)C</smiles>

B.

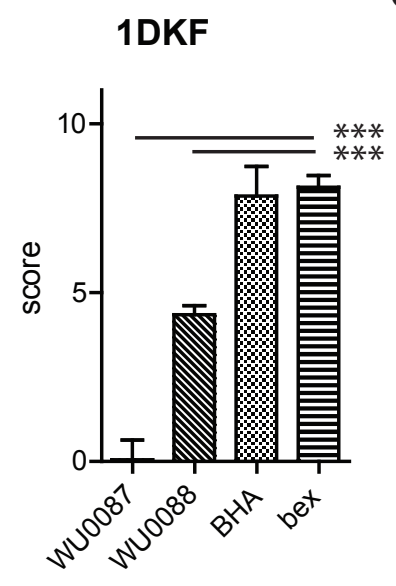

C.

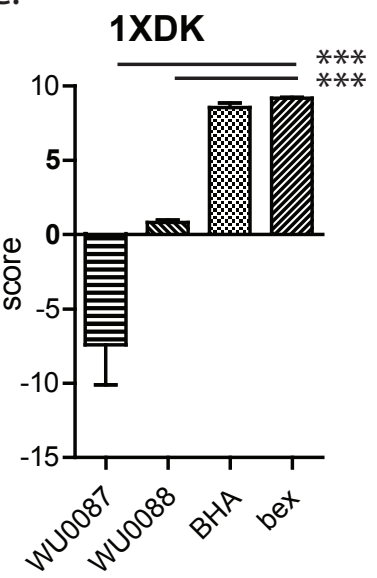

D.

\section{Gal4-RXRA}

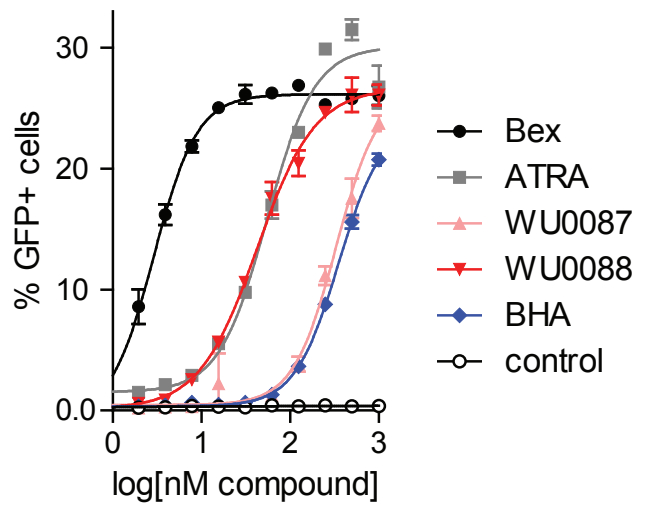

E.

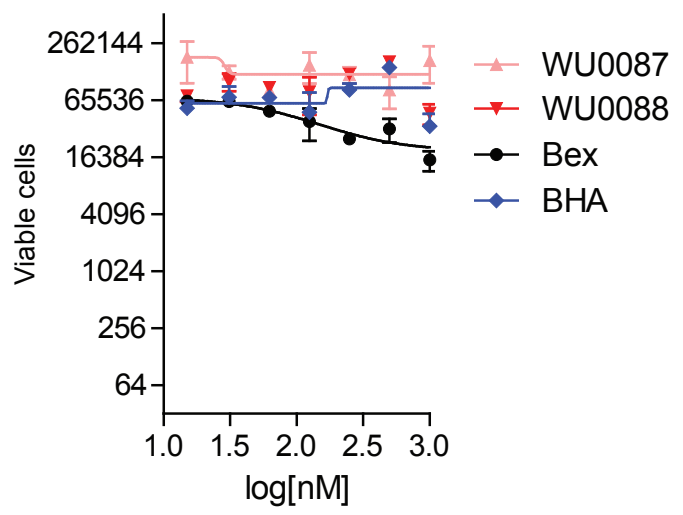

F.

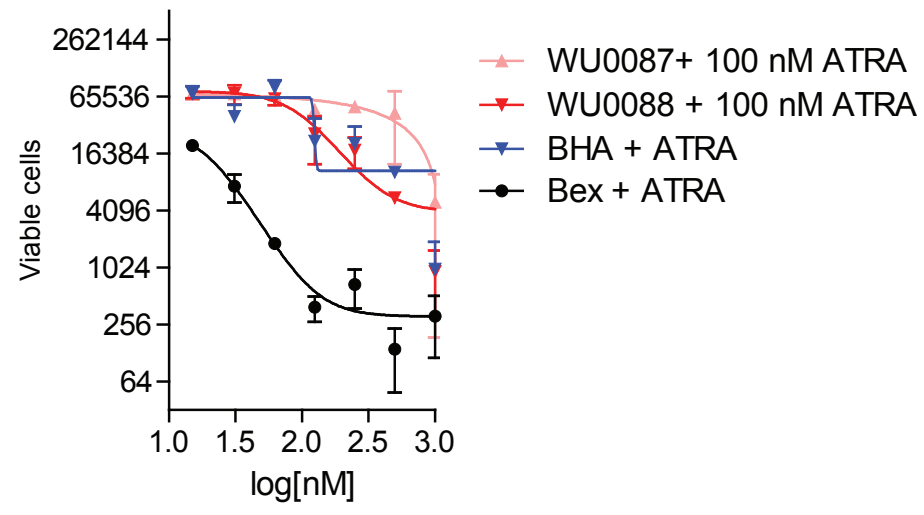

Figure 4. 
bioRxiv preprint dol: https://doi.org/10.1101/2021.05.17.444475; this version posted May 17, 2021. The copyright holder for this preprint (which was not certified by peer review) is the author/funder, who has granted bioRxiv a license to display the preprint in perpetuity. It is made available under aCC-BY-NC-ND 4.0 International license.

A.

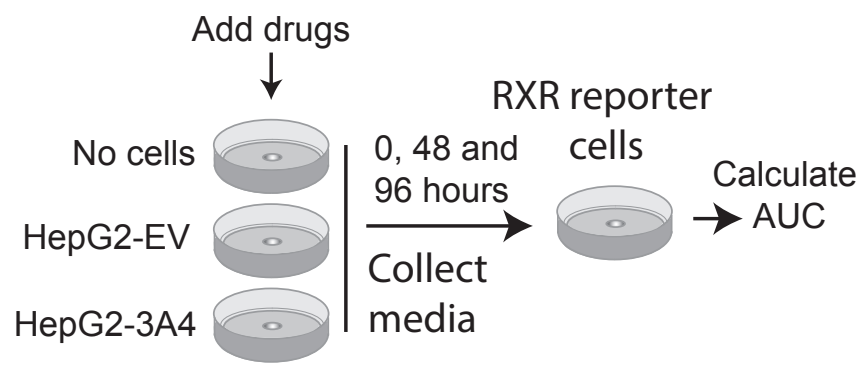

B.

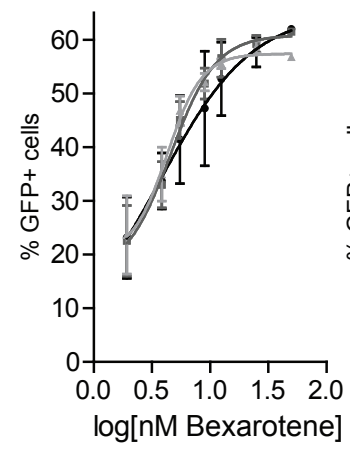

HepG2-EV

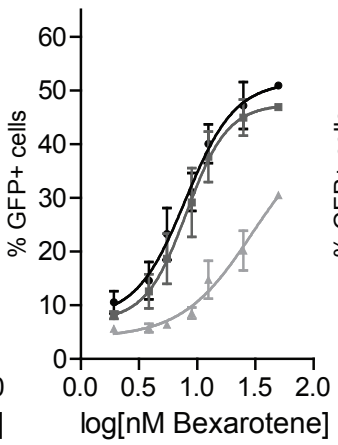

HepG2-3A4

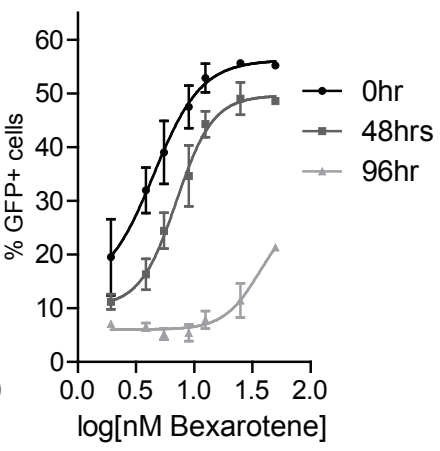

C.

No cells

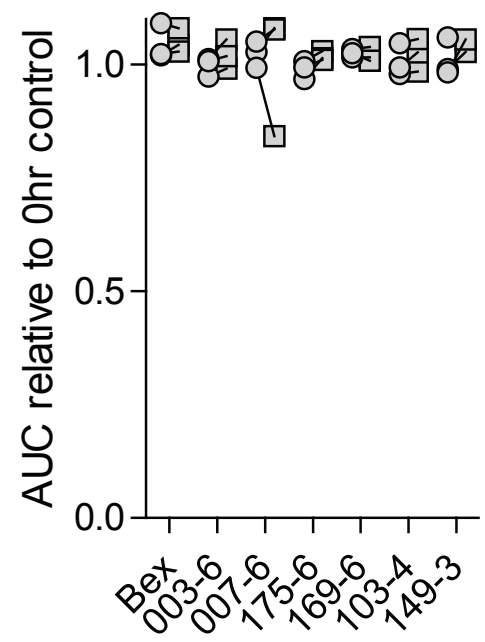

D.

Hep G2-EV

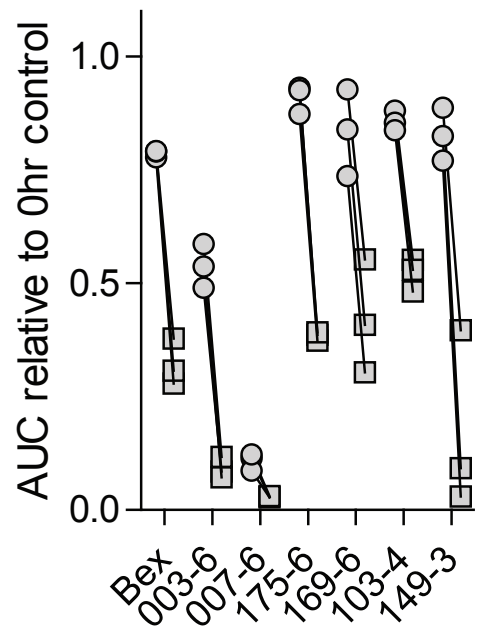

E.

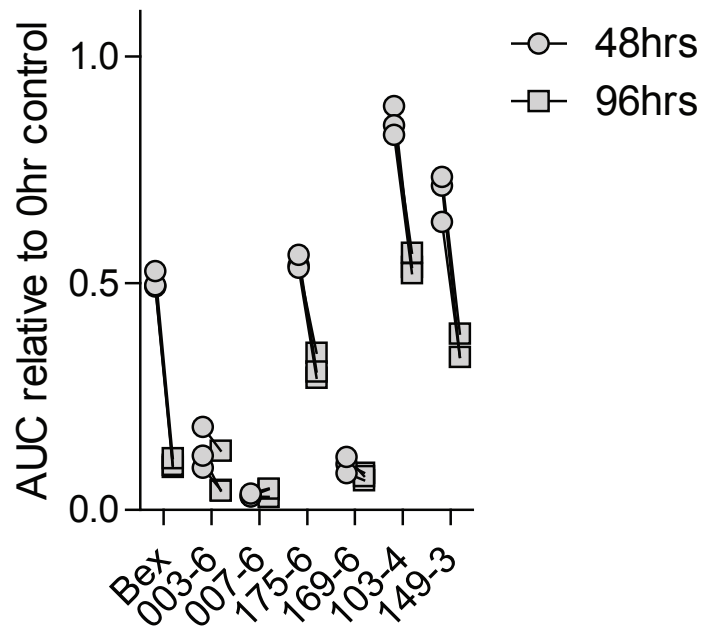

F.

Bexarotene 003-6<smiles>C=C(c1ccc(C(=O)O)cc1)c1cc2c(cc1C)C(C)(C)CCC2(C)C</smiles><smiles>Cc1cc2c(cc1C1(c3ncc(C(=O)O)cn3)CC1)C(C)(C)CC2(C)C</smiles>

$175-6$<smiles>C=CCN(c1ccc(C(=O)O)cn1)c1cc2c(cc1C)C(C)(C)COC2(C)C</smiles><smiles>C=C(c1ncc(C(=O)O)cn1)c1cc2c(cc1C)C(C)(C)CC2(C)C(=O)O</smiles><smiles>CCN(c1ccc(C(=O)O)cn1)c1cc2c(cc1C)C(C)(C)COC2(C)C</smiles>

103-4<smiles>C=C(c1ccc(C(=O)O)c(F)c1)c1cc2c(cc1C)C(C)(C)C=CC2(C)C</smiles>

149-3<smiles>C=C(c1ccc(C(=O)O)cc1)c1cc2c(cc1C)C(C)(C)C=CC2(C)C</smiles>

Figure 5. 